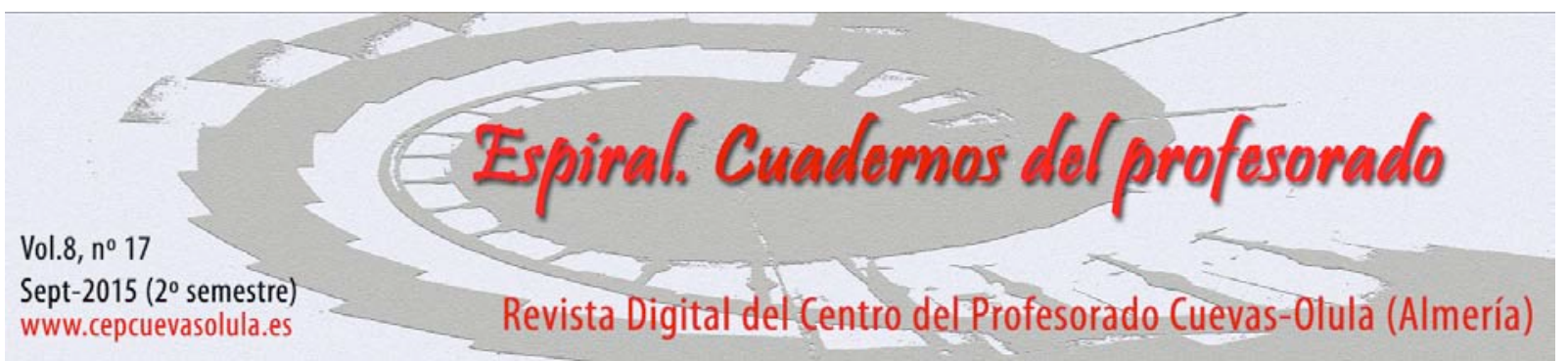

\title{
SYMBALOO COMO PUERTA DE ACCESO A LAS TECNOLOGÍAS DE LA INFORMACIÓN Y LA COMUNICACIÓN EN LA ENSEÑANZA DE ÁLGEBRA
}

\author{
Symbaloo as gateway to information and communications technology in teaching \\ algebra
}

\section{María del Mar Calles Burgos}

IES Abyla, Ceuta, España

\begin{abstract}
RESUMEN: Este artículo reflexiona sobre las relaciones entre las nuevas tecnologías, la motivación estudiantil y el empleo de metodologías alternativas en las aulas. La infinidad de recursos educativos en Internet y la escasa inclusión de los mismos en la enseñanza han sido los puntos de partida de esta iniciativa. El presente estudio plantea la introducción de las TIC en el campo matemático del álgebra, a un grupo de 55 estudiantes de $2^{\circ}$ E.S.O., como alternativa metodológica a las clases tradicionales. Durante el desarrollo del proceso se guió a los estudiantes en la realización de actividades, visualización de vídeos y participación en juegos educativos relacionados con el álgebra en niveles incipientes. La experiencia se ha llevado a cabo haciendo uso de la plataforma Symbaloo, de forma que en todo momento los estudiantes podían acceder a los distintos recursos y ser así partícipes de su propio ritmo de aprendizaje. El análisis de resultados desvela un alto índice de motivación por parte de un alumnado que tradicionalmente ha venido mostrando síntomas de apatía y de abandono escolar. El artículo concluye identificando los elementos valorados por los propios estudiantes en la motivación escolar.
\end{abstract}

Palabras clave: Educación, motivación, Symbaloo, TIC, álgebra.

ABSTRACT: This article reflects on the relationship amongst new technologies, student motivation and the use of alternative methodologies in classrooms. The numerous educational resources on the Internet and the poor inclusion of them for teaching have been the starting point of this initiative. This study raises the introduction of ICT in the mathematical field of algebra with a group of 55 students from 2nd ESO, as a methodological alternative to traditional classes. During the development process students were guided in performing activities, watched videos and participated in educational games related to emerging algebra levels. The experience was carried out with the use of Symbaloo platform, so that at all time the students had access to various resources and thus participant at their own learning pace. The analysis of results reveals a high level of motivation of a student body that traditionally has been showing signs of apathy and dropout. The article.

Key words: Education, motivation, Symbaloo, ICT, algebra 
Calles Burgos, M. M. (2015). Symbaloo como puerta de acceso a las tecnologías de la información y la comunicación en la enseñanza de álgebra. Espiral. Cuadernos del Profesorado, 8(17), 35-45. Disponible en: http://www.cepcuevasolula.es/espiral.

Fecha de recepción: 26/06/2015

Fecha de aceptación: 18/07/2015
Enviar correspondencia a: mar_calles@hotmail.com

\section{1.- INTRODUCCIÓN}

El bajo rendimiento académico de los estudiantes de matemáticas junto al mal uso que éstos hacen de su tiempo libre es uno de las preocupaciones que acompaña en la mayoría de las ocasiones a los profesionales de la enseñanza de esta materia. Ante esta situación, el proyecto plantea aprovechar el potencial de las Tecnologías de la Información y la Comunicación (TIC) para motivar al alumnado en el aprendizaje y facilitar el proceso de adquisición de determinadas competencias en el área de Matemáticas; en concreto nos centraremos en el campo del álgebra.

Estudios recientes demuestran que no se aprovecha al máximo el potencial de las tecnologías para apoyar el proceso educativo (Azumendi, 2005) y cómo la investigación educativa se ha centrado principalmente en las actividades de aprendizaje dentro de las aulas (Sawyer, 2006). No debemos permanecer pasivos ante esta brecha digital abierta entre alumnos(as) y profesores(as). Es, por tanto, deber de todos los profesionales que trabajamos en el ámbito de la enseñanza, renovarnos tecnológicamente e incluir de una manera efectiva las TIC en nuestro día a día.

Los cambios a los que está sujeta la sociedad en la que vivimos reflejan la necesidad de transformar el aprendizaje de los alumnos de forma que reforcemos su autonomía personal y su capacidad para aprender en diversos contextos. En esta línea, Aprender a Aprender se convierte en una herramienta imprescindible para garantizar la capacitación y autonomía de las personas a lo largo de sus vidas. En general se viene aceptando la explicación que Hautamäki et al. (2002) han aportado:

- Aprender a aprender se define como la competencia de y la disposición a adaptarse a tareas novedosas.

- Competencia, por definición, se refiere a la aplicación de procedimientos generales y de lo que ya se conoce, a nuevas situaciones y tareas.

- La disposición se refiere a varios subsistemas de motivación y actitudinales; ambos relacionados con uno o una misma y con el contexto.

- Aprender a aprender se desarrolla mediante buenas prácticas educativas.

Las TIC tienen una gran importancia en cuanto al desarrollo de esta competencia, que requiere la creación de situaciones de enseñanza que promuevan el desarrollo de nuevas destrezas, la automatización de la información previamente disponible y la toma de conciencia del alumnado de sus progresos y dificultades en el aprendizaje. La Figura 3 muestra un mapa conceptual de las TIC como soporte al aprendizaje.

Es importante resaltar que, tal y como afirma Elena Martín (2008), para que el alumno tenga sensación de competencia es preciso que tenga éxito en las tareas que se le plantean. Esto significa que hay que ajustar la actividad al nivel de aprendizaje del alumnado. Si el docente plantea una actividad demasiado complicada es probable que el alumno no consiga realizarla y piense que no es capaz de aprender.

Ahora bien, es obvio que esta capacidad para el aprendizaje continuo y autónomo va a depender mucho de habilidades relacionadas con la búsqueda, filtrado y tratamiento de la información. En este sentido aparece el concepto "Entornos Personales de Aprendizaje (PLE)". Podemos afirmar que todos tenemos un entorno personal a través del cual aprendemos. Dicho entorno puede estar formado por personas (familiares, amigos, compañeros de trabajo,...) o por objetos tecnológicos (televisión, cine, radio,...); por lo tanto, lo que está cambiando es el contexto. 
En la actualidad son muchos los autores que investigan sobre los PLE, ligándolos a la importancia de las TIC y sus aplicaciones a la enseñanza. Entre ellos citaré los diversos trabajos realizados por Tur et al. (2014), dedicados a las aplicaciones de las Nuevas Tecnologías en la Educación.

Trouche (2000) y Lagrange (2000) desarrollaron un marco conceptual para un enfoque instrumental en el aprendizaje de las matemáticas, en ambientes basados en TIC. Por su parte, Ramirezparis (2009) propone usar alternativas lúdicas en la enseñanza de las matemáticas, para ayudar a los alumnos(as) con dificultades a superar las mismas. Väljataga y Laanpere (2010) aseguran que el PLE permite a las personas actualizar continuamente sus conocimientos y habilidades en un mundo cada vez más interconectado y mediado tecnológicamente.

Un PLE consta de herramientas y estrategias de búsqueda de información, siendo necesario que esta información se organice y se realice una metacognición y autorregulación de lo aprendido. Ahora bien, todos los tipos de herramientas que conforman el PLE del alumno(a) deben ser organizadas y para ello, Urbina (2013) nos presentan las páginas de inicio como una herramienta de ayuda para organizar la información.

En el campo del Álgebra diversos autores como Ventura (Universidad Michoacana de México), Acosta (Universidad Nacional de Argentina) o Reverte (Universidad de las Islas Baleares) han realizado numerosos estudios sobre la influencia de las TIC en el aprendizaje del álgebra. De los múltiples estudios realizados en esta línea la mayoría de ellos destacan los siguientes puntos en el empleo de las TIC aplicadas a la enseñanza:

- Elevan la motivación y mejoran la comunicación de los estudiantes.

- Mejoran la eficacia comunicativa entre profesores(as) y alumnos(as), al crearse un diálogo que permite romper las barreras espacio-temporales.

- El aprendizaje se interioriza de un modo más eficiente y duradero.

- Creación de una atmósfera cálida para el aprendizaje.

- Mejora en la organización del conocimiento, de forma que permiten crear interrelaciones entre los distintos conceptos.

La motivación constituye, así mismo, uno de las principales pilares de la educación. Un estudiante motivado se involucra y concentra más en la clase y ello favorece el aprendizaje (Passey et al. 2004, en Condie y Munro, 2007). Aún más, la experiencia de algunos programas de informática educativa ha mostrado que el aumento de la motivación de los estudiantes por el uso de las TIC en clases aumenta el nivel de asistencia al colegio (Borthwick y Lobo, 2005). Se ha demostrado que la naturaleza visual de algunas tecnologías involucra más a los estudiantes y refuerza la comprensión de conceptos (HMIE, 2005; Livingstone y Condie, 2003; Passey, 2004; citados en Condie y Munro, 2007).

En Matemáticas (Cox et al., 2003) han demostrado que animaciones y simulaciones refuerzan la comprensión de conceptos y la creación de abstracciones que mediante métodos tradicionales no son posibles. Algunos de los estudios realizados (López, 2010) muestran que el uso de "Scratch" ayuda a desarrollar habilidades de resolución de problemas, especialmente destrezas como la descomposición de los mismos. Este lenguaje de programación, heredero del popular "Logo" de los años 80 aprovecha los cambios tecnológicos ocurridos desde entonces para incluir una interfaz más atractiva.

Las TIC, además, permiten a los estudiantes con pocas destrezas simbólicas y numéricas desarrollar estrategias para poder resolver situaciones problemáticas. Si bien, integrar las TIC en clase de Matemáticas y en concreto en el aprendizaje del Álgebra, implica redefinir la forma que aprendemos y enseñamos la materia (Hodges y Conner, 2011). Debemos decidir cuáles son los recursos apropiados para conseguir las competencias que deseamos desarrollar en el alumnado.

\footnotetext{
${ }^{1}$ Scratch es un entorno de programación creado en Massachusetts Institute of Technology (MIT) como resultado de la investigación llevada a cabo en el Lifelong Kindergarten Group.
} 
Aunando los puntos principales de interés de los párrafos anteriores, surge esta experiencia, cuyo eje principal es motivar al alumnado e incentivarle a desarrollar la competencia de Aprender a Aprender. El entorno elegido para promover estas actitudes ha sido la página de inicio Symbaloo, a través de la cual el alumno(a) descubrirá nuevas habilidades de aprendizaje de forma autónoma y lúdica.

Symbaloo es una plataforma on-line, que nos permite gestionar nuestro propio PLE, utilizando un interfaz sencillo e intuitivo. La aplicación se basa en el uso de un Webmix (escritorio) o un conjunto de ellos. Cada uno de estos escritorios, tiene la posibilidad de albergar hasta 60 bloques también llamados ítems, a través de los cuales podemos acceder a las distintas direcciones webs que nos interesen.

Durante el desarrollo del proyecto se han planteado las siguientes preguntas de investigación:

1. ¿El uso de ambientes enriquecidos por las TIC aumenta el interés de los alumnos hacia el aprendizaje del álgebra?

2. ¿Es Symbaloo una herramienta adecuada para la organización de los recursos en el aprendizaje del álgebra?

3. ¿Este tipo de metodología me permite adaptarme mejor a las necesidades de los alumnos(as)?

Estos interrogantes, a su vez, nos han llevado a definir el objetivo general de la presente investigación: diseñar y evaluar una experiencia de aprendizaje del álgebra en un entorno adaptado a las TIC, que se concreta en cuatro objetivos específicos:

- Objetivo 1: Analizar el uso de Symbaloo como herramienta de apoyo para la integración de recursos y materiales de aprendizaje.

- Objetivo 2: Determinar el grado de motivación del alumnado hacia el aprendizaje de las matemáticas.

- Objetivo 3: Contrastar la autopercepción del alumnado en el aprendizaje adquirido.

- Objetivo 4: Analizar las cualidades de las herramientas TIC existentes para el aprendizaje del álgebra en niveles incipientes.

Se pretende en todo momento facilitar el aprendizaje de esta área y conseguir una mejor predisposición hacia la materia, mediante el uso de herramientas TIC. Este hecho, además, permitirá mejorar la Competencia Digital y la Competencia para Aprender a Aprender en nuestro alumnado.

\section{2.- METODOLOGÍA}

Teniendo en cuenta las características de los distintos tipos de investigación, ésta se desarrolla bajo un enfoque cualitativo, en tanto que busca la identificación y la reflexión sobre los fenómenos que enfrentan a la persona consigo misma. Los puntos que sustentan este enfoque cualitativo en la metodología del presente proyecto son varios. Entre ellos podemos destacar los siguientes:

- Es un proyecto inductivo: en el que partimos de experiencias determinadas para extraer conclusiones generales.

- Es un proyecto en el que utilizamos los datos obtenidos sin incorporar nuestro conocimiento previo.

- Es un proyecto que estudia la realidad de un centro educativo concreto.

Este estudio centra su investigación en un instituto de enseñanza secundaria de la ciudad autónoma de Ceuta. Los alumnos(as) son en un alto porcentaje de origen magrebí, provenientes de familias de bajos recursos económicos y bajo nivel educativo. La mayoría de ellos, al ser preguntados por su futuro profesional, no se plantean su acceso a la universidad ni a ningún tipo de estudios superiores.

En un análisis más detallado del alumnado sobre el que hemos realizado el proyecto, se observa que la mayoría presenta dificultades para resolver problemas de la vida cotidiana que requiera 
el uso de operaciones matemáticas. Son estudiantes a los que les cuesta planificar su trabajo y, en general consideran muy complicada la asignatura, lo que se refleja en un bajo rendimiento y motivación.

La experiencia se ha llevado a cabo en dos grupos específicos de $2^{\circ}$ curso de E.S.O. con características muy similares. La media de alumnos por clase es de 27 estudiantes, estando varios de ellos diagnosticados como alumnos de necesidades educativas específicas (ACNEE). Del total de alumnos de la muestra el $53 \%$ son varones, frente al $47 \%$ restante que son mujeres, todos ellos con edades comprendidas entre los 14 y los 17 años.

La estrategia de investigación planteada en este proyecto ha llevado a los docentes implicados a un cambio de actitud, planteándose una seria reflexión acerca del grado de interés y de compromiso en el proceso de enseñanza y aprendizaje. Éste revulsivo culminó imaginando cuál podría ser una solución coherente y factible a los problemas planteados, formulando la propuesta de un plan de actuación y finalmente, llevándola a la práctica.

Durante la implementación del proyecto se ha hecho uso de una de las aulas de informática del I.E.S., equipada con 14 ordenadores. Al comienzo de cada una de las clases los estudiantes debían abrir su Symbaloo, accesible desde la siguiente dirección: http://iesabyla.symbaloo.com/. Sin embargo, y al haber utilizado una versión de prueba, la cuenta de Symbaloo educativo ya ha sido cancelada y el enlace anterior ha dejado de estar disponible.

Cada uno de los días programados para la realización de la actividad ha sido diseñado en su propio Webmix. En todos ellos aparece un bloque con el enlace al archivo de la actividad programada para ese día. Los alumnos deben leer la descripción de dicha actividad, visualizar las páginas Web seleccionadas y practicar con los diversos ejercicios propuestos on-line. En la Tabla 1 se muestra un cronograma del desarrollo de las sesiones y actividades del proyecto.

Tabla 1. Cronograma de las sesiones.

\begin{tabular}{|c|c|c|c|c|c|}
\hline & \multicolumn{5}{|c|}{ ABRIL 2015} \\
\hline & 14 & 17 & 21 & 24 & 28 \\
\hline $\begin{array}{l}\text { CLASE } 1 \\
\text { Explicación de la actividad. Toma de contacto con el proyecto y con } \\
\text { la plataforma Symbaloo }\end{array}$ & & & & & \\
\hline $\begin{array}{l}\text { CLASE } 2 \\
\text { Lenguaje algebraico }\end{array}$ & & & & & \\
\hline $\begin{array}{l}\text { CLASE } 3 \\
\text { Monomios. Operaciones con monomios }\end{array}$ & & & & & \\
\hline $\begin{array}{l}\text { CLASE } 4 \\
\text { Polinomios. Operaciones con polinomios }\end{array}$ & & & & & \\
\hline $\begin{array}{l}\text { CLASE } 5 \\
\text { Productos notables. Operaciones con productos notables }\end{array}$ & & & & & \\
\hline
\end{tabular}

En todo momento se ha recurrido a la observación directa de los alumnos, así como a otros instrumentos específicos de recogida de información, como son un diario de clase, un test de conocimientos al finalizar cada una de las sesiones (tal y como se muestra en la Figura 5) y un cuestionario tipo test proporcionado a todo el alumnado al finalizar el proyecto de investigación (parcialmente mostrado en la Tabla 2).

A continuación de describirán de forma más detallada cada uno de estos instrumentos:

- Diario de clase: es un recurso que se emplea para recoger información directamente de los implicados en el proceso formativo o para registrar por él mismo la evolución de un proceso o el desarrollo de una acción (Barroso y Cabero, 2010).

Mediante la confección de este diario de clase se pretender analizar las siguientes categorías: el funcionamiento de la herramienta Symbaloo, la detección de problemas técnicos, las destrezas de los alumnos, la capacidad de trabajar con los diferentes recursos, la competencia digital del alumnado, 
la motivación de los mismos y, finalmente el uso de los recursos específicos de aprendizaje empleados.

Tabla 2. Fragmento del Cuestionario Final perteneciente al bloque de ítems

"Motivación y Desarrollo Personal".

\begin{tabular}{|c|c|c|c|c|c|}
\hline $\begin{array}{l}\text { 8. Me gustaría que en otras asignaturas se hicieran } \\
\text { experiencias similares con el uso de las TIC. }\end{array}$ & TD & $\mathrm{D}$ & I & $\mathrm{A}$ & TA \\
\hline $\begin{array}{l}\text { 9. Esta forma de trabajar me ha hecho sentir protagonista de } \\
\text { mi proceso de aprendizaje. }\end{array}$ & TD & $\mathrm{D}$ & I & A & TA \\
\hline 10. Me ha gustado esta forma de realizar las clases. & TD & $\mathrm{D}$ & I & A & TA \\
\hline $\begin{array}{l}\text { 11. Me lo he pasado mejor con esta forma de realizar las } \\
\text { actividades propuestas. }\end{array}$ & TD & $\mathrm{D}$ & I & A & TA \\
\hline
\end{tabular}

- Test de Evaluación: para conocer el impacto del entorno enriquecido con TIC, los alumnos(as) realizaban al finalizar cada una de las clases los Test de Conocimientos creados para ese día en concreto. Cada una de estas pruebas se correspondía a los contenidos practicados durante dicha sesión en al aula de informática.

Las pruebas están formadas por una media de $6-7$ preguntas tipo test, incluyendo algunas de ellas 2 ó 3 preguntas de conocimientos en las que no se les proporciona alternativa de elección a los alumnos(as). Han sido realizadas para responderlas con una duración estimada de 5 minutos.

Con esta herramienta se pretende medir el nivel de conocimientos adquiridos durante la clase $\mathrm{y}$, al mismo tiempo, motivar al alumnado en el seguimiento y realización de actividades. Este instrumento de recogida de información se ha considerado determinante en esta experiencia, dada las características del alumnado que lo realizó.

- Cuestionario Final: al finalizar el proyecto y para evaluar el grado de consecución de los objetivos, se les realizó un cuestionario tipo test a los alumnos(as) implicados.

El cuestionario está formado por un total de 21 ítems, 17 de los cuales se corresponden a preguntas cerradas tipo Likert con cinco respuestas (TD, D, I, A, TA) y los cuatro restantes a preguntas abiertas en las que los alumnos(as) participantes pueden expresar su opinión y aportar sus propios puntos de vista. Las siglas anteriores se corresponden a los siguientes significados: TD (totalmente en desacuerdo); D (en desacuerdo), I (indiferente); A (de acuerdo); TA (totalmente de acuerdo). La Tabla 3 muestra el objeto de estudio de cada uno de los ítems empleados en el cuestionario, agrupados por categorías.

Tabla 3. Categorías del Cuestionario Final.

\begin{tabular}{|c|c|c|}
\hline ITEMS & DIMENSIÓN & OBJETO DE ESTUDIO \\
\hline $\begin{array}{c}{[1-3]} \\
\& \\
{[14-17]}\end{array}$ & Contenidos de álgebra & $\begin{array}{l}\text { Son preguntas enfocadas al aprendizaje del álgebra y al } \\
\text { uso de las TIC como herramientas facilitadoras de dicho } \\
\text { aprendizaje. }\end{array}$ \\
\hline 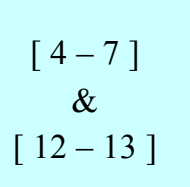 & $\begin{array}{c}\text { Proceso de } \\
\text { enseñanza-aprendizaje }\end{array}$ & $\begin{array}{l}\text { Son preguntadas dirigidas a averiguar si este tipo de } \\
\text { experiencia ha llevado a los alumnos(as) a mejorar su } \\
\text { relación con la materia, a acercarse más a ella, a dedicarle } \\
\text { más tiempo, a disfrutar haciendo las actividades. }\end{array}$ \\
\hline$[8-11]$ & $\begin{array}{c}\text { Motivación } \\
\text { y } \\
\text { desarrollo personal }\end{array}$ & $\begin{array}{l}\text { Son preguntas dirigidas a conocer si los alumnos(as) se } \\
\text { habían divertido con la experiencia y les gustaría } \\
\text { continuar trabajando en esta línea. }\end{array}$ \\
\hline
\end{tabular}




\section{3.- RESULTADOS Y DISCUSIÓN}

Las características de los diferentes instrumentos han requerido un tipo de estudio diferente para cada uno de ellos, tal y como se muestra en los distintos apartados que lo configuran:

- Resultados extraídos del Diario: del análisis del diario llevado a cabo durante la experiencia se extraen bastantes resultados, entre los que destacaré los siguientes:

- Problemas técnicos: aparte de algunos incidentes sin demasiada importancia, el último día de proyecto se cortó la conexión con Internet y el $2^{\circ}$ de los grupos no pudo finalizar la clase con normalidad. También se constató que el número de ordenadores fue claramente insuficiente para el número de alumnos.

- Competencia digital y Destreza en el uso de Symbaloo: los alumnos mostraron escasas destrezas en el uso de Internet y de los ordenadores pero siguieron las actividades sin dificultad debido a la sencillez de los recursos utilizados.

- Acceso a cuentas propias: el primer día de clase estaba planteada la creación de cuentas de correo electrónico y el posterior uso de Google Drive como herramienta de trabajo colaborativo. Sin embargo, y debido a numerosos problemas en su creación, finalmente trabajamos sin este recurso.

- Motivación: los alumnos se mostraron claramente motivados y con un alto grado de curiosidad hacia los nuevas actividades propuestas.

- Resultados extraídos de los Test de Evaluación: el estudio de dichos test se ha realizado de manera individualizada y pormenorizada para cada uno de ellos, calculando los porcentajes de aciertos a las distintas preguntas planteadas. Para facilitar el estudio de los mismos se han volcado dichos datos en una página Excel, representándolos mediante diagramas de sectores. A modo de ejemplo, la Figura 2, muestra el diagrama de sectores correspondiente a una de las preguntas tipo test formulada durante la sesión dedicada a los monomios.

Con objeto de obtener una visión conjunta de este instrumento de recogida de datos se han fundido los resultados de los diferentes Test de Conocimientos en un único diagrama de barras, mostrado en la Figura 1. El diagrama refleja la evolución de los alumnos(as) en el número de aciertos en dichos tests. El eje de las Y se corresponde al porcentaje de preguntas acertadas, que aunque en todo momento mantiene unos niveles bastante

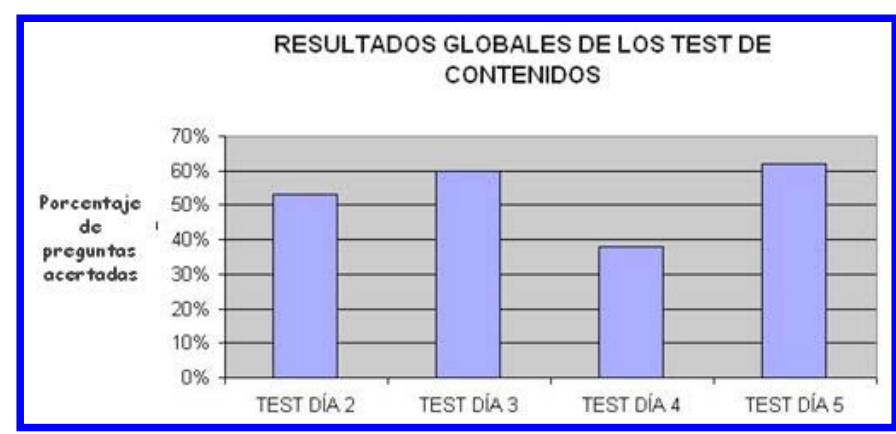

Figura 1. Resultados de los Test de Evaluación. bajos, presenta un ligero ascenso en todos los días, excepto el día 4. Esto puede ser debido al empleo de los polinomios durante ese día, y la dificultad que estos conllevan.

- Resultados extraídos del Cuestionario Final: el cuestionario final fue respondido por un total de 45 alumnos y han sido analizadas cada una de sus preguntas de forma individual, así como agrupadas por bloques de contenidos.

- Con respecto al primer bloque de contenidos (aprendizaje de álgebra) llama la atención que sólo uno de de los estudiantes ha respondido negativamente a la pregunta "Me ha resultado sencillo usar Symbaloo", mientras que el resto del grupo afirma resultarle fácil o muy fácil.

- Del segundo bloque de contenidos (mejora en el aprendizaje debido a las TIC) se desprende una aproximación positiva por parte de los estudiantes a la materia. Un elevado número de alumnos(as) ha valorado positivamente la realización de ejercicios 
propuestos desde Symbaloo, así como el gusto por utilizar dicha plataforma en el aprendizaje de otras asignaturas.

- El último bloque de contenidos (motivación) refleja que la experiencia les ha resultado gratificante y motivadora. En este bloque todas las preguntas, a excepción de una de ellas ("Esta forma de trabajar me ha hecho sentir protagonista de mi proceso de aprendizaje") reflejan una clara motivación en el alumnado hacia el uso de las TIC en la práctica docente.

Finalmente, tal y como se refleja en la Figura 4, se ha realizado un estudio de forma conjunta recurriendo a gráficos de diagramas de sectores; de dicho estudio se desprende un alto grado de satisfacción en los tres bloques de contenidos tratados por parte del alumnado.

Tal y como reflejan los resultados de este estudio el uso de las TIC en el desarrollo de la práctica docente es altamente satisfactorio. Múltiples estudios realizados en los últimos años confirman este hecho. Este enlace muestra la ponencia de la profesora de matemáticas $\mathrm{M}^{\mathrm{a}}$ José Rey, bajo el título "Jugando con las ecuaciones".

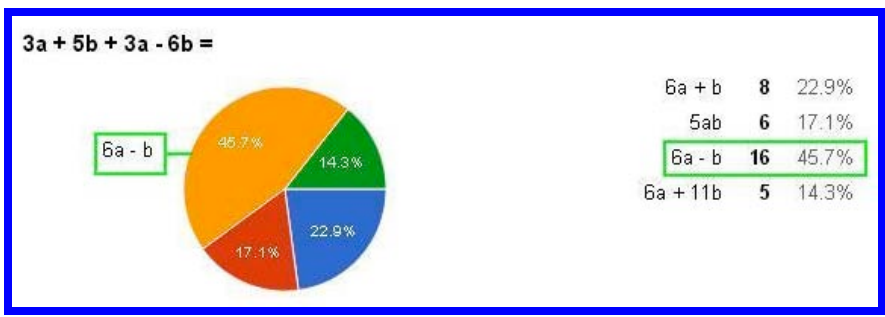

Figura 2. Diagrama de sectores correspondiente a los resutlados de una de las preguntas del Test de Evalución correspondientes a la sección "Monomios"

Destacaré igualmente el trabajo

realizado por García y Romero (2009) en el que estudian la influencia de las nuevas tecnologías en el aprendizaje y las actitudes matemáticas en el aula de secundaria. Del estudio se desprende que, si bien las transformaciones cognitivas fueron leves, se produjo una mejora notable en las actitudes hacia las matemáticas debido al uso de las TIC, demostrando el alumnado un mayor espíritu crítico, creatividad y aumento en la flexibilidad de pensamiento. En esta misma línea las TIC también produjeron un aumento en la motivación y en la mejora del comportamiento de los alumnos(as).

Las experiencias anteriores no son más que una simple muestra de los múltiples ejemplos del uso de las nuevas tecnologías en la enseñanza que podemos encontrar en Internet.

\section{4.- CONCLUSIONES}

Tras la exposición de todos estos puntos, ha llegado el momento de comentar las conclusiones extraídas de este estudio. Para ello, se han utilizado los 3 instrumentos de recopilación de información descritos anteriormente $\mathrm{y}$, de forma paralela, se ha realizado una triangulación de los resultados obtenidos en los mismos.

De todo esto se infieren las siguientes conclusiones:

- La satisfacción del alumnado en el uso de las TIC y su interés por el aprendizaje del álgebra en este tipo de contexto es altamente satisfactorio. La actitud de los alumnos(as) hacia una determinada asignatura se forja a través de muchos años y es difícil modificarla. Una experiencia tan corta como esta no pretende modificar esta percepción pero sí un acercamiento positivo hacia la misma y un nuevo enfoque de la materia.

- La propuesta de uso de Symbaloo en este proyecto ha resultado acertada, tanto por su facilidad de uso, su interfaz amigable, las posibilidades que ofrece a nivel educativo en el que nos encontramos, la posibilidad inmediata de crear bloques nuevos y, de esta manera, permitir a los alumnos(as) su propia participación en su proceso de aprendizaje, etc.

- Finalmente, y respecto a si este tipo de metodología permite adaptarse mejor a los distintos ritmos de aprendizaje no he podido obtener conclusiones específicas. Siendo unos de los motivos el tiempo empleado en la realización del proyecto (que ha sido muy limitado), así como al factor novedad, que ha supuesto este tipo de metodología para los chicos(as). 


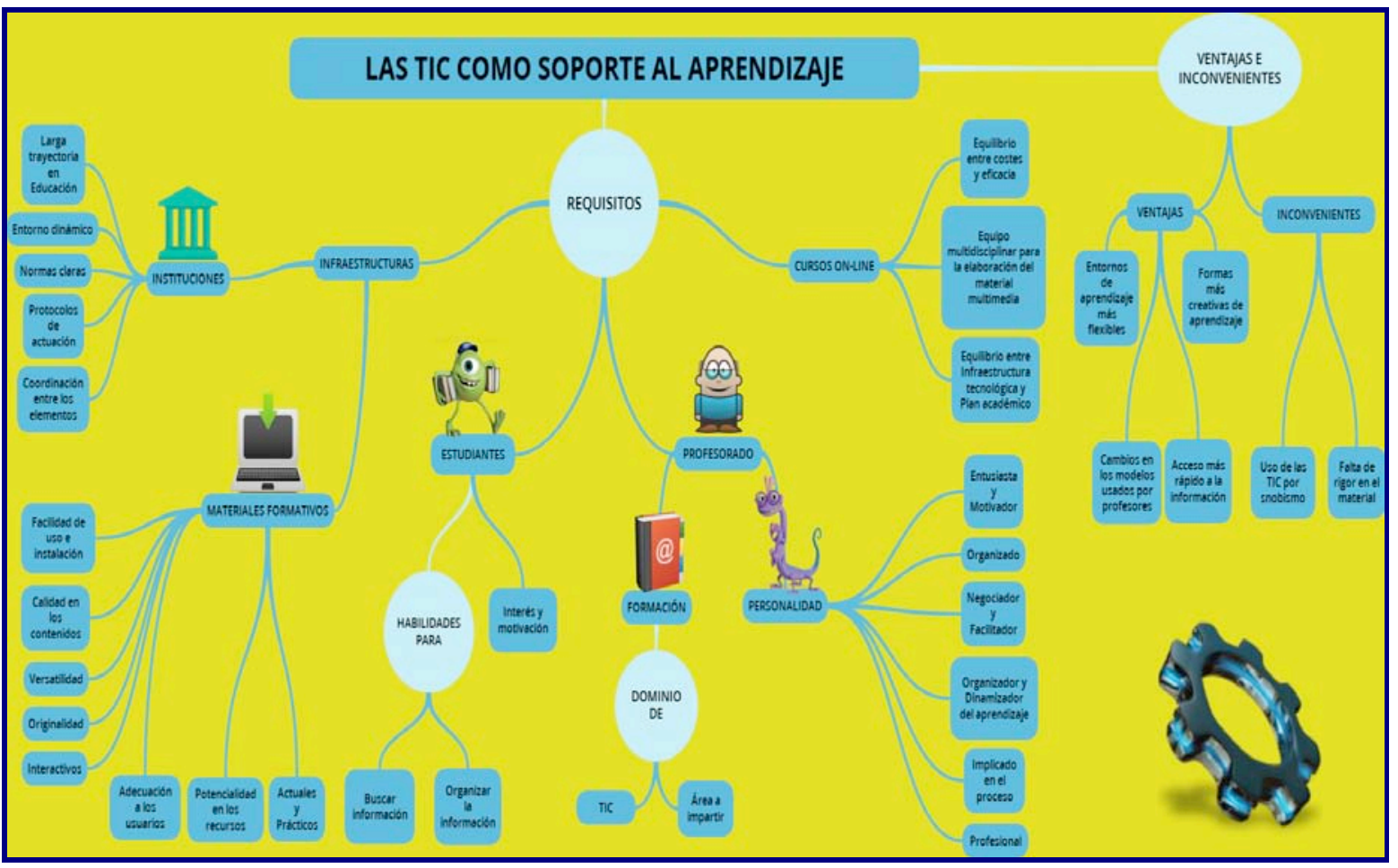

Figura 3. Mapa conceptual de las TIC como soporte al aprendizaje. Fuente: Elaboración propia.

\section{MEJORAS Y FUTURAS LIINEAS DE INVESTIGACIÓN}

Por último me gustaría comentar que todo proyecto de investigación es susceptible de mejoras, abriendo caminos a nuevas líneas de investigación. En este sentido, los errores detectados por parte del docente (tanto en el desarrollo del proyecto y su puesta en funcionamiento), como por parte del alumnado (en el trabajo a diario), deberían ser utilizados como trampolín para futuros proyectos.

La experiencia llevada a cabo se ha mostrado incapaz de prestar apoyo suficiente a aquellos alumnos(as) con necesidades educativas específicas o con distintos ritmos de aprendizaje. El descubrimiento de una nueva actividad, dado el carisma innovador que para ellos presentaba este proyecto, se imponía siempre frente al aprendizaje de los pasos previos. Se plantea así la posibilidad de usar este tipo de herramientas potenciando la capacidad de adaptación a las necesidades que presenten los alumnos(as).

Con respecto a la tipología de actividades a realizar, se ha detectado que no se debe abusar de las actividades largas y de excesiva dificultad, pues hacen que los alumnos(as) pierdan interés y motivación por la materia. La inclusión de un mayor número de actividades grupales y que fomenten 
la competitividad también puede ser considerada como una propuesta de mejora para ocasiones posteriores. Este tipo de actividades fomentan el trabajo colaborativo y hacen que los propios alumnos manifiesten un mayor interés al tener que "enfrentarse" a sus compañeros.

Este proyecto abre nuevas líneas de investigación en las que poder seguir trabajando para mejorar el aprendizaje de las matemáticas y la motivación del alumnado. La inclusión de las TIC como herramienta de apoyo al aprendizaje y elemento potenciador de la motivación, abre un amplio campo de estudio.

Una posible línea sobre la que profundizar el estudio es la gamificación. Han sido muchos los alumnos(as) que han mostrado su interés por uno de los juegos (la pista algebraica) planteados en el desarrollo de las sesiones y mencionado que les habría gustado el empleo de estrategias similares para mejorar el aprendizaje.

También cabe destacar, entre las respuestas de los alumnos(as) la importancia que muchos de ellos han dado al hecho de trabajar en parejas. Sería excelente la realización de un proyecto de recopilación/creación de recursos de trabajo colaborativo para el aprendizaje de las matemáticas.

Para finalizar, pero no por ello menos importante, se puede profundizar en los PLEs y su relación con la gestión del aprendizaje por

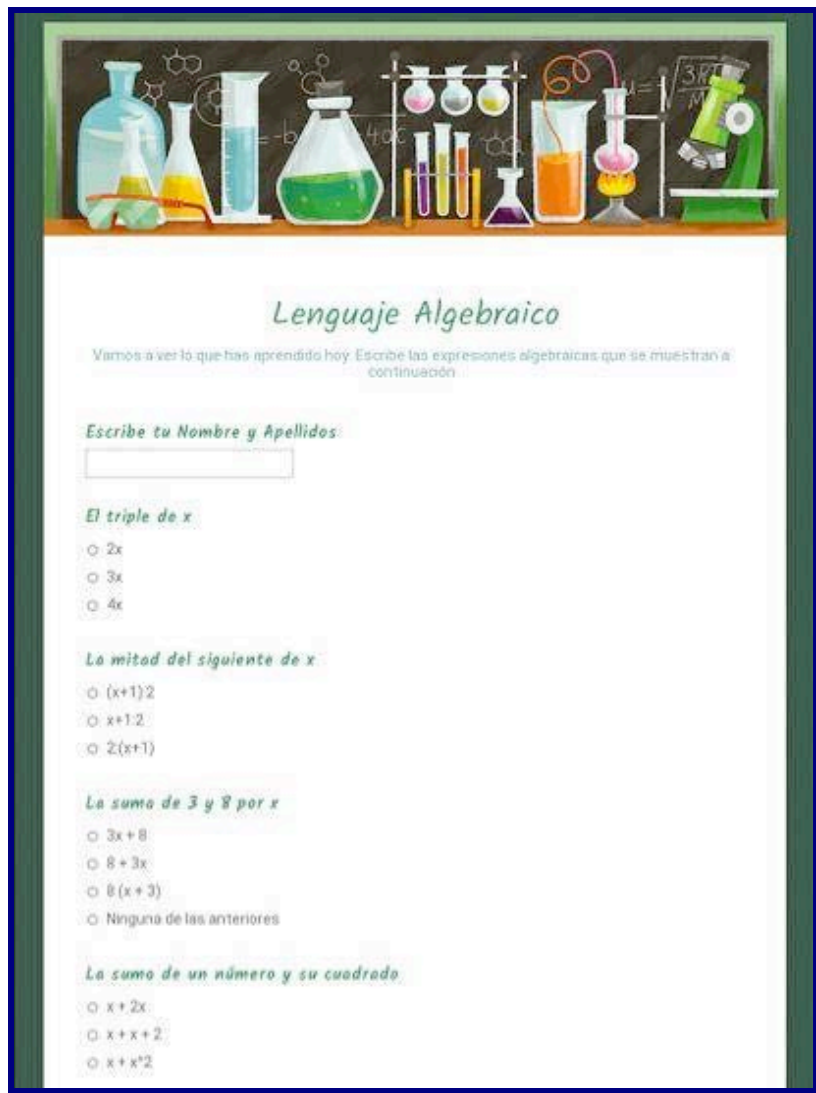

Figura 5. Fragmento del Test de Conocimientos perteneciente a la sección "Lenguaje algebraico".

parte del alumnado. Sería interesante hacer una comparativa de los mismos para identificar aquellos que mejor se adapten a la enseñanza de las matemáticas en los distintos niveles educativos.

\section{6.- REFERENCIAS}

Azumendi, E. (2005). Educación constata el escaso uso de las nuevas tecnologías en los colegios. Disponible en: http://elpais.com/diario/2005/04/11/paisvasco/1113248401_850215.html

Barroso, J. \& Cabero, J. (2010). La investigación educativa en TIC. Visiones prácticas. Madrid: Síntesis.

Borthwick, A. \& Lobo, I. (2005). Lessons from Costa Rica. Learning and Leading with Technology, 33(2), 1821.

Brophy, J. (1998). Motivating students to learn. Boston, McGraw-Hill.

Condie, R., Munro, B., Muir, D., \& Collins, R. (2005). The Impact of ICT Initiatives in Scottish Schools: Phase 3. Edinburgh: SEED. http://www.scotland.gov.uk/Publications/2005/09/14111116/11170

Cox, M., Abbott, M. W., Blakeley, T., Beauchamp, T., \& Rho, V. (2003). ICT and attainment: A review of the research literature ICT in Schools. Research and Evaluation Series, 17. DfES-Becta. Disponible en: http://www.becta.org.uk/page_documents/research/ict_attainment_summary.pdf.

García, M. M. \& Romero, I.M. (2009). Influencia de las nuevas tecnología en la evolución del aprendizaje y las actitudes matemáticas de estudiantes de secundaria. Electronic Journal of Research in Educational Psychology, 7(17), 369-396. Disponible en: http://investigacionpsicopedagogica.org/revista/articulos/17/espannol/Art_17_306.pdf 
Hautamäki, J., Arinen, P., Eronen, S., Hautamäki, A., Kupiainen, S., Lindblom, B. et al. (2002). Assessing Learning-to-Learn: a Framework (pp. 277-291). Helsinki: National Board of Education.

Hautamäki, J., Arinen, P., Eronen, S., Hautamäki, A., Kupiainen, S., Lindblom, B. et al. (2002). Assessing Learning-to-Learn: a Framework, 277-291. Helsinki: National Board of Education.

Hodges, T. E. \& Conner, E. (2011). Crafting Roles: Reflections from a Teacher and a Student in a Technology Rich Mathematics Classroom. Mathematics Teacher, 104(6), 432 - 438.

López, M. \& Gomá, A. (2010). Scratch, un entorno agradable para la programación. III Seminario sobre actividades para estimular el talento precoz en Matemáticas. Facultad de Psicología. Universidad de Valencia.

Martín, E. (2008). Aprender a aprender: clave para el aprendizaje a lo largo de la vida. Participación Educativa, $9,72-78$.

Ramirezparis, X. (2009). La lúdica en el aprendizaje de las matemáticas. Revista del Instituto de Estudios en Educación, 10, 2-8.

Sawyer, R.K. (2006). The Cambridge Handbook of the Learning Sciences. Cambridge, MA: Cambridge University Press.

Tur, G., Marín, V., Moreno, J. \& Urbina, S. (2014). Disponible en: Going beyond the diagrams: student teachers' reflections on their PLE. The PLE Conference 2014.

Urbina, S., Arrabal, M., Conde, M., \& Ordinas, C. (2013). Las páginas de inicio como herramienta de ayuda para organizar el PLE. Un análisis comparativo. EDUTEC, Revista Electrónica de Tecnología Educativa, 43. Disponible en: http://edutec.rediris.es/Revelec2/Revelec43/paginas inicio herramientas organizar PL E analisis.html

Väljataga, T. \& Laanpere, M. (2010). Learner control and personal learning environment: a challenge for instructional design. Disponible en: https://www.mendeley.com/catalog/learner-control-personallearning-environment-challenge-instructional-design/ 